\title{
Recuperação da dimensão vertical de oclusão com prótese temporária overlay: relato de caso
}

\author{
Recovery of vertical dimension of occlusion with temporary overlay denture: case report \\ Recuperación de la dimensión vertical de oclusión con prótesis temporales overlay: reporte de caso \\ Halysson Ferreira CÉZAR ${ }^{1}$ \\ Franklin Barbosa da SILVA ${ }^{2}$

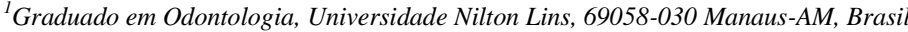 \\ ${ }^{2}$ Doutorando em Saúde Pública, Universidade de São Paulo, 01246-904, São Paulo-SP, Brasil. Professor de Prótese Total e Oclusão, \\ Curso de Odontologia, Universidade Nilton Lins, 69058-030 Manaus-AM, Brasil
}

\begin{abstract}
Resumo
A dimensão vertical de oclusão é descrita como a altura do terço inferior da face enquanto existe o máximo de contatos posteriores entre os dentes, e, sua diminuição é ocasionada pela perda total ou parcial de dentes posteriores. O objetivo do presente trabalho é relatar um caso clínico de restabelecimento da dimensão vertical de oclusão através da confecção de prótese parcial removível overlay. Paciente compareceu à clínica odontológica da Universidade Nilton Lins queixando-se da aparência do seu rosto, aspecto do sorriso, dentes desgastados e dificuldade de alimentar-se. Ao exame extraoral, observou-se aprofundamento do sulco-nasogeniano, diminuição de altura do terço inferior da face e ausência de pontos de gatilho. Ao exame intraoral constatou-se extensa reabsorção óssea no arco inferior, ausência de todos os dentes posteriores do arco inferior, e, a presença de desgastes e fraturas dentárias em ambos os arcos. Após os exames, constatou-se a presença de desarmonia oclusal e diminuição da dimensão vertical de oclusão. Foram realizados planejamento e enceramento de diagnóstico com o objetivo de restabelecer a oclusão ideal perdida. Optou-se então pela confecção de próteses temporárias removíveis overlay com encaixe sobre a estrutura dentária remanescente. $\mathrm{O}$ tratamento executado mostrou-se satisfatório, pois devolveu as funções e altura do terço inferior da face perdidas, sendo capaz de preparar o paciente e o sistema estomatognático para execução do procedimento reabilitador definitivo.
\end{abstract}

Descritores: Dimensão Vertical; Ajuste Oclusal; Perda de Dente, Prótese Parcial Temporária.

\begin{abstract}
The vertical dimension of occlusion is described as the height of the lower third of the face while there is the maximum of posterior contacts between the teeth, and its decrease is caused by the total or partial loss of posterior teeth. The objective of the present study is to report a clinical case of restoration of the vertical dimension of occlusion through the manufacture of removable temporary overlay denture. Patient attended the dental clinic of Nilton Lins University complaining about the appearance of his face, smile appearance, worn teeth and difficulty feeding. Extraoral examination revealed deepening of the nasolabial sulcus, decreased height of the lower third of the face and absence of trigger points. Intraoral examination revealed extensive bone resorption in the lower arch, absence of all posterior teeth of the lower arch, and the presence of dental wear and fractures in both arches. After the examinations, the presence of occlusal disharmony and the loss of vertical occlusion dimension were observed. Diagnostic planning and waxing were performed with the objective of restoring lost ideal occlusion. We then chose to make removable overlapping temporary prostheses with a fitting over the remaining dental structure. The treatment performed proved to be satisfactory, as it restored the functions and height of the lower third of the face, being able to prepare the patient and the stomatognathic system to perform the definitive rehabilitation procedure.
\end{abstract}

Descriptors: Vertical Dimension; Occlusal Adjustment; Tooth Loss; Denture, Partial, Temporary.

\section{Resumen}

La dimensión vertical de oclusión se describe como la altura del tercio inferior de la cara mientras existe el máximo de contactos posteriores entre los dientes, y su disminución es ocasionada por la pérdida total o parcial de dientes posteriores. El objetivo del presente trabajo es relatar un caso clínico de restablecimiento de la dimensión vertical de oclusión a través de la confección de prótesis parcial removible overlay. El paciente asistió a la clínica odontológica de la Universidad Nilton Lins quejándose de la apariencia de su cara, aspecto de la sonrisa, dientes desgastados y dificultad para alimentarse. En el examen extraoral, se observó profundización del surco-nasogeniano, disminución de altura del tercio inferior de la cara y ausencia de puntos de gatillo. En el examen intraoral se constató una extensa reabsorción ósea en el arco inferior, ausencia de todos los dientes posteriores del arco inferior, y la presencia de desgastes y fracturas dentales en ambos arcos. Después de los exámenes, se constató la presencia de desarmonía oclusal y disminución de la dimensión vertical de oclusión. Se realizaron planificación y encerado de diagnóstico con el objetivo de restablecer la oclusión ideal perdida. Se optó entonces por la confección de prótesis temporales removibles overlay con encaje sobre la estructura dental remanente. El tratamiento realizado se mostró satisfactorio, pues devolvió las funciones y altura del tercio inferior de la cara perdidas, siendo capaz de preparar al paciente y el sistema estomatognático para la ejecución del procedimiento rehabilitador definitivo.

Descriptores: Dimensión Vertical; Ajuste Oclusal; Pérdida de Diente; Dentadura Parcial Provisoria.

\section{INTRODUÇÃO}

A perda dos elementos dentários ocasiona diversas complicações no funcionamento do sistema estomatognático, altera de forma significativa a mastigação, fonética e deglutição, gerando também possíveis desgastes dentários, alterações na estética, perda da harmonia do sorriso, aumento do sulco nasogeniano, tensões nos músculos da mastigação e problemas na articulação temporomandibular (ATM), como estalidos e travamentos ${ }^{1-3}$. Além destas, outra grave consequência é a perda da dimensão vertical de oclusão (DVO), sendo definida como a altura do terço inferior da face enquanto existe o máximo de contato posterior, o que define a correta relação oclusal $^{3-5}$. Essa alteração pode ocorrer por perda total ou parcial de dentes posteriores ${ }^{6,7}$, causando um aumento no espaço funcional livre, oclusão traumática, sobrecarga da ATM, mudanças na postura da cabeça e pescoço e possíveis desgastes dentais nos remanescentes ${ }^{8}$.

Antes do processo de reabilitação da DVO o paciente deve ser avaliado de forma minuciosa, afim de desenvolver um correto diagnóstico e melhor elaboração do plano de tratamento ${ }^{9}$. Os exames extra orais são de grande importância, dentre eles a palpação da ATM e musculaturas da face, observando se existem lesões, assimetrias faciais e 
alterações estéticas. Os exames complementares como radiografias panorâmicas e tomografias são fundamentais para avaliar o estado de saúde óssea do paciente, possibilidade de dentes inclusos e possíveis lesão não detectáveis no exame clínico ${ }^{10}$.

O principal objetivo da reabilitação da DVO é proporcionar ao paciente uma condição que restabeleça todas as funções perdidas, fazendo com que se recupere a correta relação maxilo mandibular e adequada harmonia facial ${ }^{1,11-13}$. Uma das opções de tratamento reabilitador do paciente seria a confecção de próteses temporárias, elas têm o intuito de confirmar a altura da reabilitação e restabelecer a DVO de uma maneira mais rápida, que nos possibilite confirmar se os itens protéticos a serem confeccionados serão apropriados ou não ao paciente $^{8}$.

A prótese temporária overlay tem uma grande importância nesse processo de reabilitação, pois ela não necessita de um tratamento clínico integrado, visto que é adaptada às estruturas dentárias remanescentes sem a necessidade de outros tratamentos especializados ${ }^{14}$. Ela restabelece as funções orais perdidas pela diminuição da DVO e apresenta grandes vantagens por ter um baixo custo e maior simplicidade na execução, como sua forma de encaixe é sobre a oclusal dos posteriores e incisais dos dentes anteriores, acaba oferecendo facilidade na remoção para higienização, contribuindo para adaptação do indivíduo a nova $\mathrm{DVO}^{14,15}$.

O objetivo deste trabalho é relatar um caso clínico de confecção de prótese parcial removível overlay para o restabelecimento da dimensão vertical de oclusão do paciente.

\section{CASO CLÍNICO}

Paciente de 45 anos, sexo masculino, compareceu à clínica odontológica da Universidade Nilton Lins, queixando-se da aparência do seu rosto torto (Figura 1), aspecto do sorriso, dentes desgastados e dificuldade de alimentar-se. Durante a anamnese, o mesmo relatou ser tabagista crônico, alcoólatra e ter sido diagnosticado com carcinoma verrucoso há oito anos. Para tratamento da lesão foi optado pela terapêutica cirúrgica, sendo submetido à exérese da lesão, juntamente com os elementos que habitavam o sítio. $\mathrm{Na}$ região, margem cirúrgica incluiu uma porção esquerda da língua e uma espessura óssea considerável da mandíbula do lado esquerdo. $\mathrm{O}$ mesmo teve acompanhamento médico por cinco anos, onde foi considerado curado, não apresentando recidivas. No exame extraoral foi verificada assimetria na região inferior esquerda da face, aproximação do mento com a ponta do nariz, desarmonia no sorriso, aprofundamento do sulconasogeniano e diminuição aparente da altura do terço inferior da face.

Ao exame clínico intraoral, observou-se na arcada superior desgastes nos dentes $11,12,13,14$, $21,22,23$ e 24 , o 25 com necessidade de exodontia por ser resto radicular e ausência dos dentes 15,18 e 28. Na arcada inferior a ausência dos dentes 31,32 , $33,34,35,36,38,41,45,46,47,48$ e desgaste excessivo nos dentes 42, 43 e 44. (Figura 2). Diante dos exames foi confirmada a diminuição da DVO do mesmo, como plano de tratamento inicial, prévio à reabilitação definitiva, foi indicado a recuperação da altura e oclusão ideal com prótese temporária removível overlay adaptada sobre os elementos remanescentes.

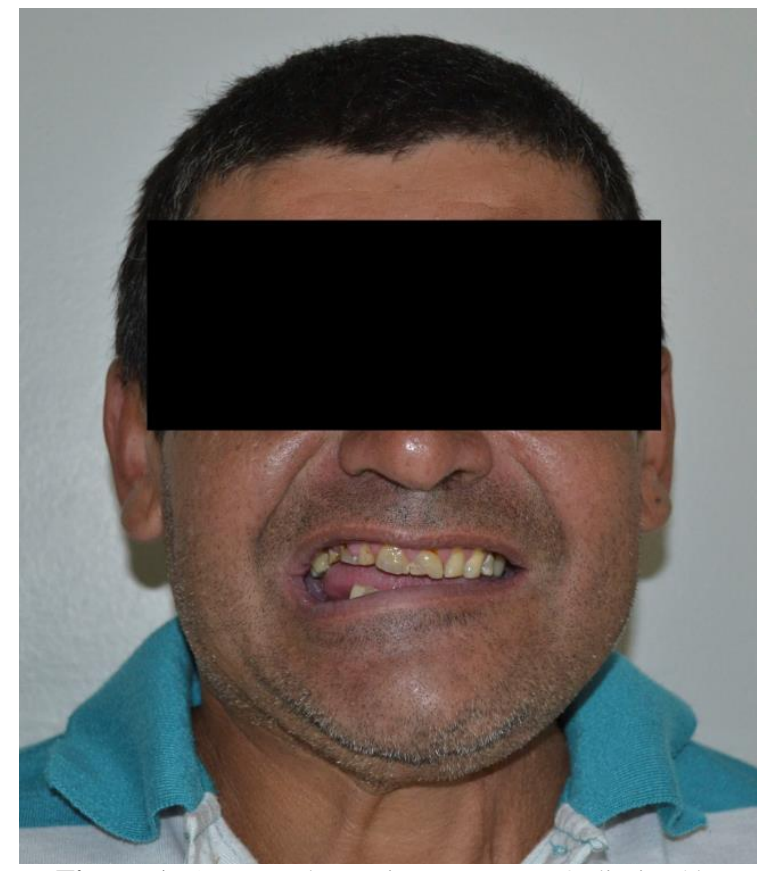

Figura 1: Aspecto do sorriso com a DVO diminuída.

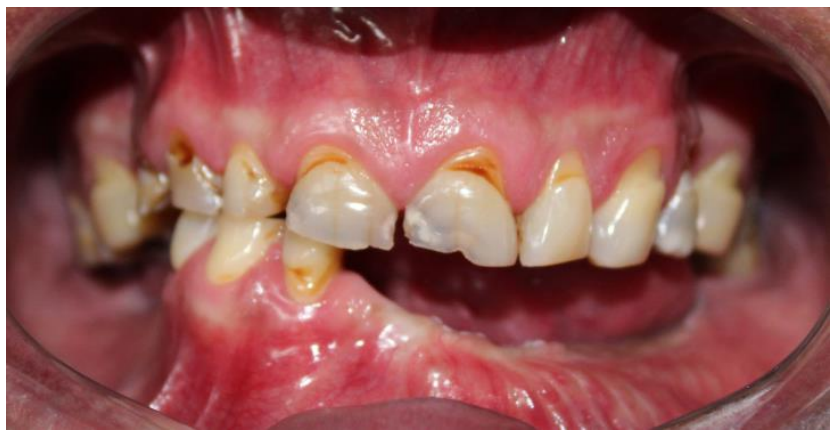

Figura 2: Diminuição da DVO.

Foi solicitada radiografia panorâmica (Figura 3), onde observou-se tratamento endodôntico insatisfatórios nos dentes 13 e 14, os mesmos foram indicados para retratamento. Iniciaram-se os procedimentos pela moldagem anatômica dos arcos superior e inferior com moldeiras metálicas perfuradas e alginato (Jeltrate, Dentsply, Brasil), obteve-se então os modelos anatômicos a partir da vazagem com gesso tipo IV (Durone, Dentsply, Brasil) (Figura 4).

$\mathrm{O}$ modelo superior foi montado em articulador semi-ajustável (ASA) (modelo 4000, Bio Art, Brasil) (Figura 5). Para o inferior foi 
confeccionada uma base de registro em resina acrílica e cera 7, com o objetivo de obter a altura próxima do considerado ideal da DVO (Figura 6).

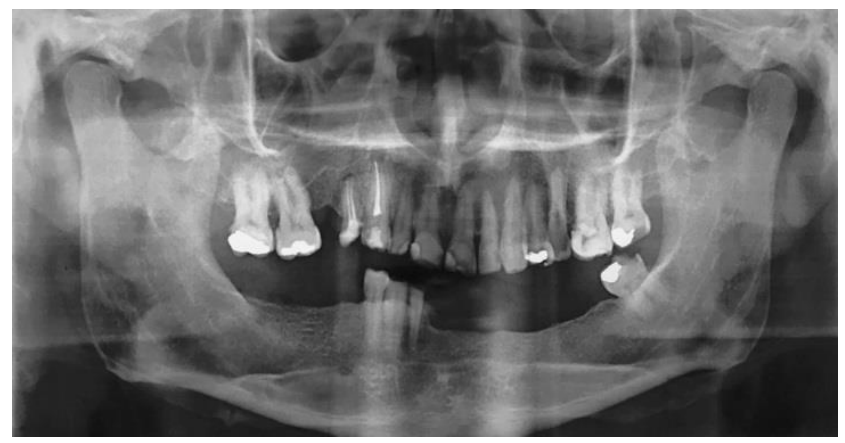

Figura 3: Radiografia panorâmica inicial.

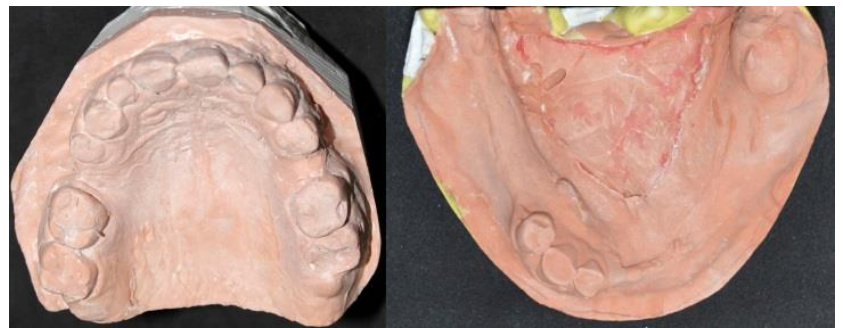

Figura 4: Modelos de estudo superior e inferior.

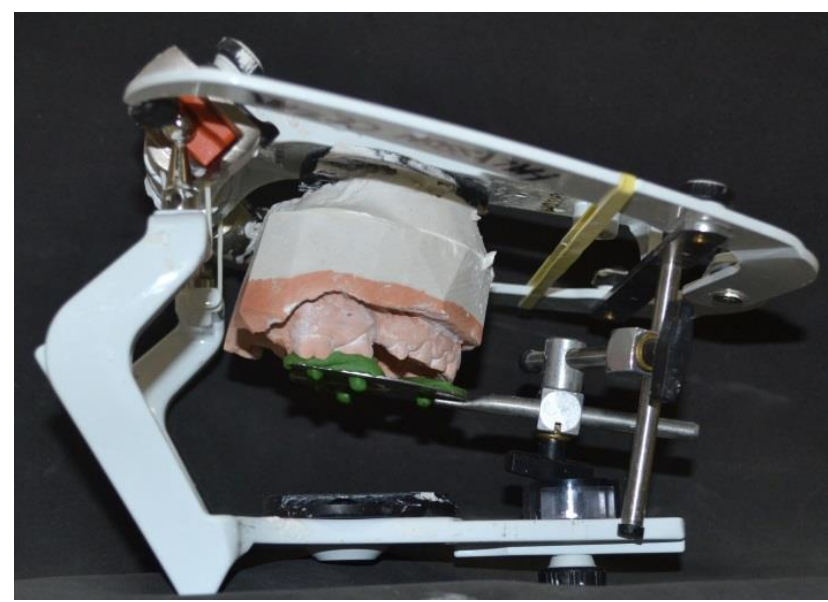

Figura 5: Modelo de estudo superior montado no ASA.

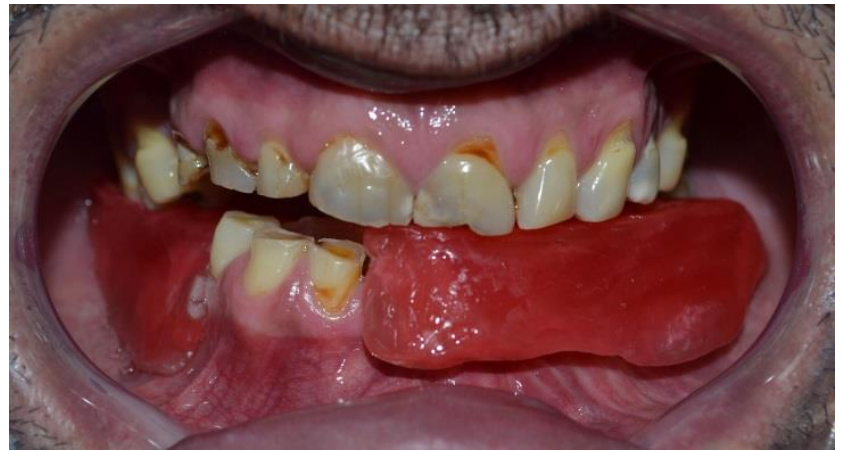

Figura 6: Placa de registro inferior.

Alguns métodos foram utilizados para restabelecer a DVO: o método métrico com auxílio do compasso de Willis, logo após, foi realizada prova estética e da proporção dos terços faciais, tendo como referência primordial a obtenção de harmonia do terço inferior da face com os demais. Estabelecida altura, o registro foi transferido para o articulador para montagem do modelo inferior.
A cor dos dentes artificiais foi selecionada através de uma escala de cores (Biotone, Dentsply) na cor 69. Após isso, foi confeccionado no modelo superior o enceramento de diagnóstico, com o auxílio da cera para escultura (Kota) foram reanatomizados os dentes desgastados respeitando o correto restabelecimento das curvas de compensação. A seguir, foram montados os dentes inferiores de estoque (Biotone, Dentsply) na base inferior já ajustada (Figuras 7, 8 e 9).

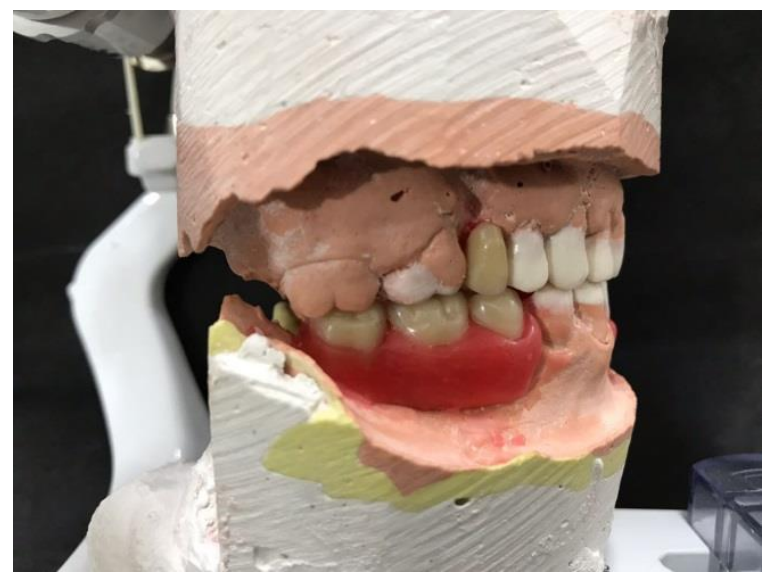

Figura 7: Vista lateral direita da oclusão estabelecida entre os modelos.

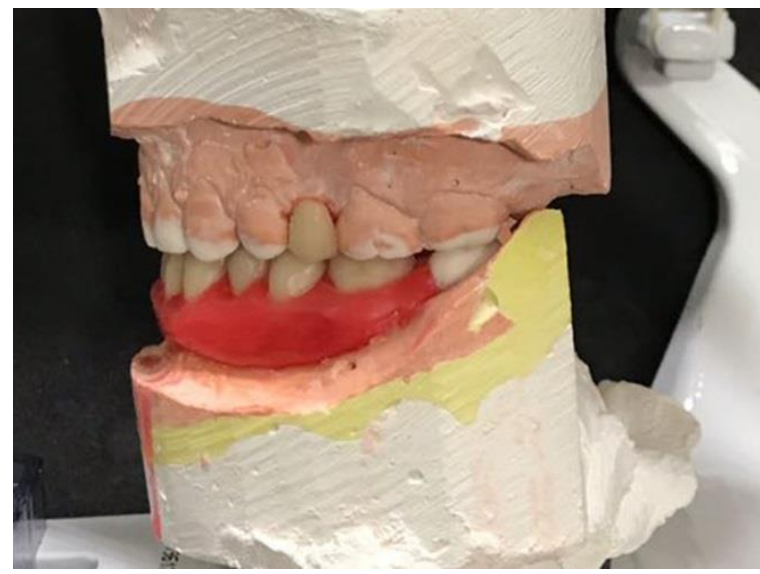

Figura 8: Vista lateral esquerda da oclusão estabelecida entre os modelos.

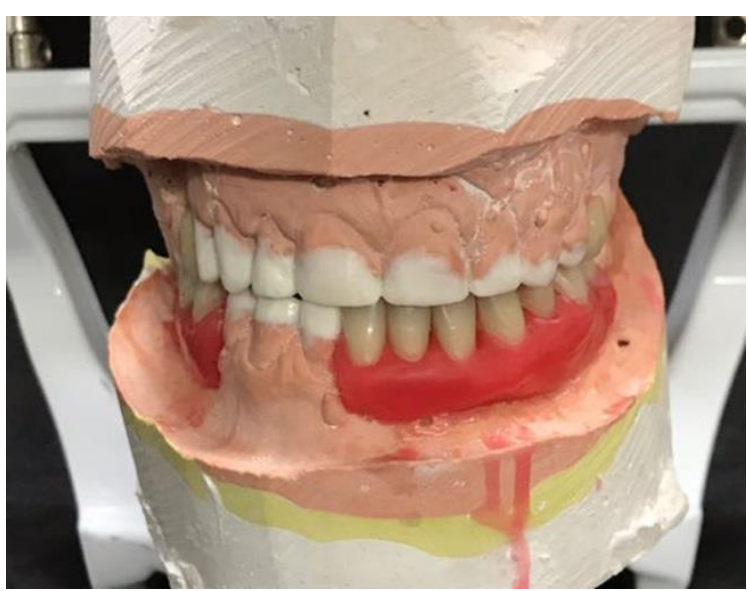

Figura 9: Vista frontal da montagem dos dentes.

Reestabeleceu-se o aspecto oclusal dos dentes anteriores, guias incisivas e de lateralidade, altura anterior e posterior, e, a máxima 
intercuspidação habitual com o máximo de contatos posteriores. Todo o restabelecimento oclusal foi realizado tomando como base o registro de altura feito em boca. No passo seguinte, foi realizada a queima da cera e na sequência, polimerização das próteses (Figuras 10, 11 e 12).

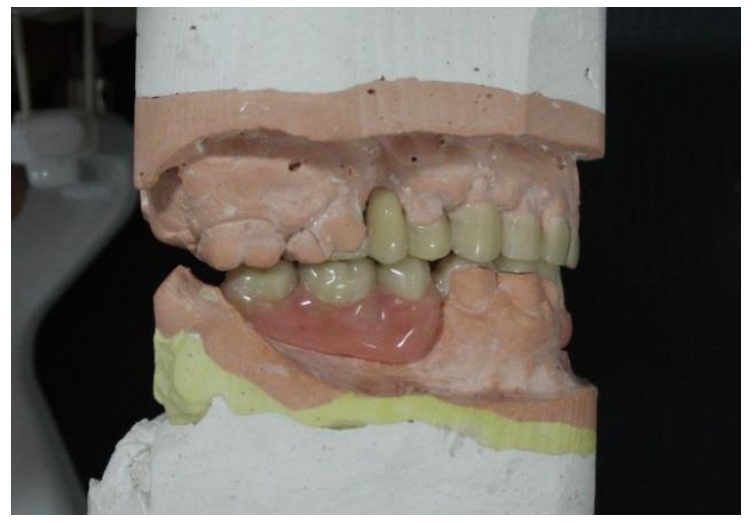

Figura 10: Vista lateral direita das próteses após polimerização.

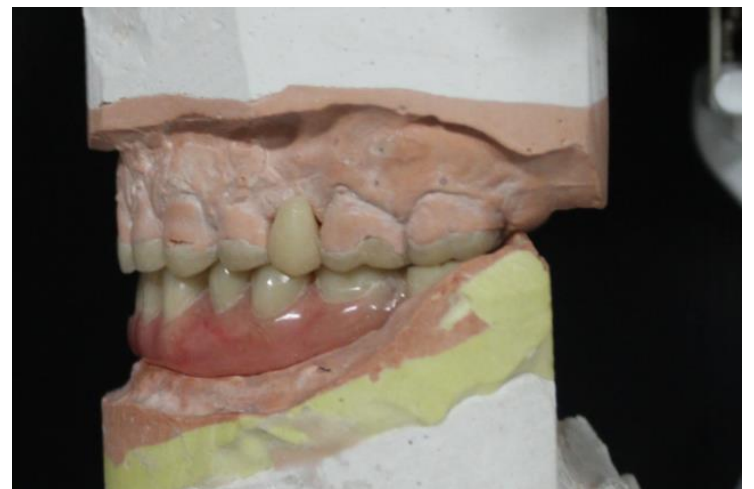

Figura 11: Vista lateral esquerda das próteses após polimerização.

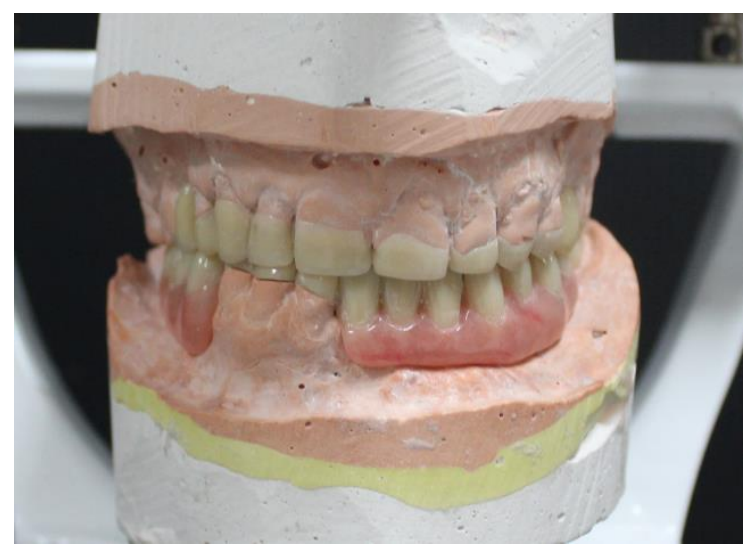

Figura 12: Vista frontal das próteses após polimerização.

No atendimento seguinte, foi feito o desgaste para adaptação das próteses superior e inferior, depois de adaptadas executou-se o reembasamento da região de encaixe sobre os dentes. Foram feitos os ajustes em oclusão cêntrica, lateralidade e protrusiva, checados os pontos de contato dos dentes com carbono (Figura 13), seguido de desgaste com broca Minicut quando necessário. Em seguida, verificou-se a nova altura estabelecida através do teste fonético de Silverman, solicitando ao paciente que pronunciasse palavras sibilantes, após isso finalmente confirmouse a nova DVO (Figuras 14 e 15).

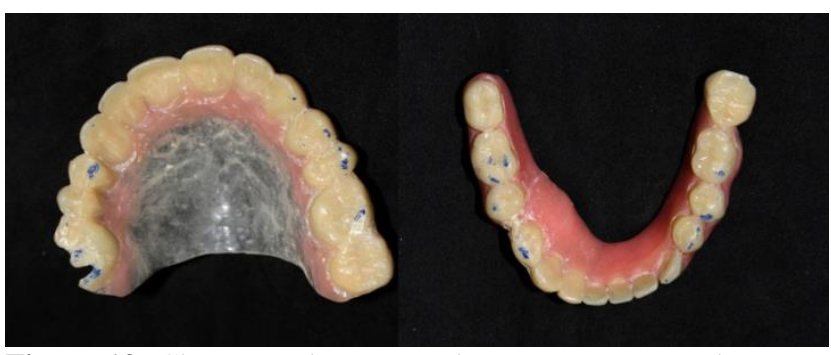

Figura 13: Checagem dos pontos de contato com o carbono nas próteses.

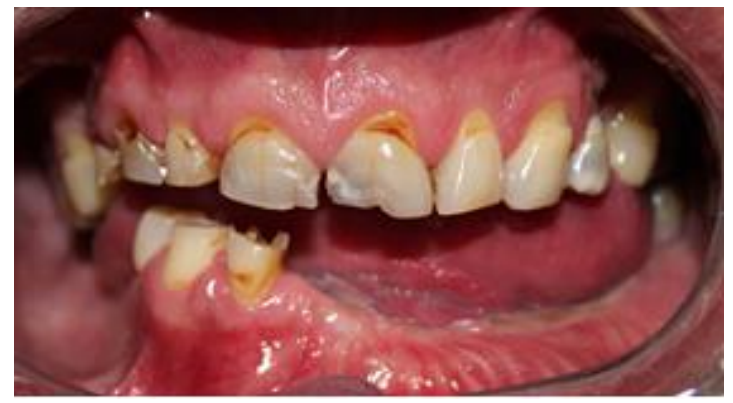

Figura 14: Aspecto inicial com a DVO diminuída.

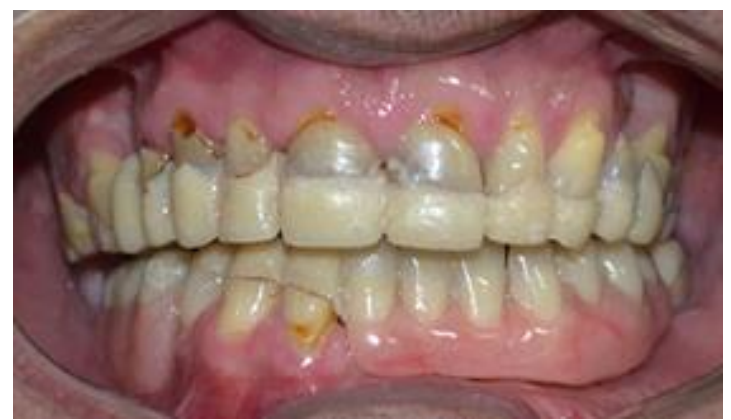

Figura 15: Aspecto final com a nova DVO reestabelecida.

Foi comunicado ao paciente que a adaptação da prótese demora normalmente de 3 a 4 semanas. Nessa fase de adequação pode ocorrer aumento de salivação, dificuldade de deglutição e dificuldade na pronúncia de algumas palavras. Nas primeiras consultas de acompanhamento, foi relatada a ocorrência de náusea e instabilidade da prótese inferior durante a mastigação. Para solução da primeira situação diminui-se o recobrimento do palato da prótese, e, para diminuir a sensação de báscula foi realizado novo reembasamento na região de encaixe. Não houve necessidade de reajuste da dimensão, pois não foi observado desconforto muscular e articular. Com o padrão de normalidade da oclusão restabelecido, pode-se dar início aos procedimentos clínicos de reabilitação.

\section{DISCUSSÃO}

Silva et al. $^{16}$ destacam que alguns cirurgiões-dentistas efetuam a reabilitação oral devolvendo aleatoriamente dentes ausentes, sem considerar as alterações inerentes aos casos, como o comprometimento de músculos, ligamentos e articulação temporomandibular. No presente caso, um adequado planejamento prévio foi feito para a recuperação de todas as funções bucais perdidas, dentre elas o correto restabelecimento da DVO, harmonia oclusal em cêntrica e excêntrica. 
Para Souza et al. ${ }^{17}$ alguns critérios devem ser considerados na seleção do material reabilitador como: diminuição da DVO, higiene oral do paciente, custo do tratamento e mudança na qualidade de vida. Cesto $^{7}$ afirma que a prótese parcial removível overlay é uma excelente escolha para a reabilitação, por ser fácil de confeccionar, pelo baixo custo e por ser um tratamento provisório eficaz. Estas alternativas foram fundamentais na escolha do tratamento, pois a prótese overlay mesmo sendo temporária, preencheu todos os requisitos necessários para reabilitação imediata do paciente.

Para Meirelles ${ }^{9}$ o enceramento tem a função de orientar o cirurgião dentista no correto diagnóstico, com objetivos reconstruir as partes desgastadas dos dentes, substituir os dentes ausentes, obter os contatos simultâneos nos dentes posteriores e ajuste das guias de desoclusão. Dessa forma, esse método é capaz de trazer uma previsibilidade do tratamento, auxiliando na retomada do equilíbrio oclusal e harmonia na execução dos movimentos mandibulares. ${ }^{5}$

Souza ${ }^{17}$ afirma que existem vantagens quanto ao uso de prótese temporária removível overlay em reabilitações orais, como proteção e condicionamento dos tecidos orais para uma futura reabilitação definitiva, restabelecimentos imediatos da harmonia facial e dimensão vertical, ganho estético, devolução da apropriada função mastigatória e a possibilidade de se realizar ajustes na dimensão, caso necessário. Estas vantagens foram comprovadas no presente tratamento, mostrando-se efetiva e preenchendo os requisitos necessários para o restabelecimento do sistema estomatognático.

No entanto, clinicamente foram observadas algumas desvantagens, como a dificuldade no encaixe da prótese nos remanescentes dentários e as náuseas recorrentes, devido ao recobrimento do palato. Outro ponto a se considerar seria o aumento considerável no tempo clínico, visto que se trata de uma prótese intermediária que necessitará ser substituída ao término do procedimento reabilitador.

\section{CONCLUSÃO}

O restabelecimento da dimensão vertical de oclusão é etapa imprescindível no tratamento reabilitador. No caso clínico exposto optou-se pela sua recuperação através da instalação de próteses temporárias overlay, seu uso demonstrou resultados satisfatórios, ao devolver as funções e altura do terço inferior da face perdidas, além de ser capaz de preparar o paciente e o sistema estomatognático para execução do procedimento reabilitador definitivo.

\section{REFERÊNCIAS}

1. Cardoso SV, Teixeira AR, Baltezan RL, Olchik RM. O impacto das alterações de deglutição na qualidade de vida de idosos institucionalizados.

Rev Kairós. 2014;17(1):231-45.
2. Cavalcanti YW, Oliveira LMC, Batista AUD. Prótese parcial removível provisória tipo overlay na reabilitação oral de paciente com colapso oclusão posterior. R Bras Ci Saúde. 2015; 19(2): 143-50.

3. Uppal S, Gupta NK, Tandan A, Dwivedi R, Gupta S, Kumar S. Comparative evaluation of vertical dimension at rest before extraction, after extraction na after rehabilitation whith complete denture - a cephalometric study. JOBCR. 2013 ; 3:73-7.

4. Abduo J. Safety of increasing vertical dimension of occlusion: a systematic review. QI. 2012;43(5):369-80.

5. Calixto LR, Bandeca MC, Andrade MF. Enceramento diagnóstico: previsibilidade no tratamento estético indireto. Rev Dent Press Estética. 2011;8(4):26-37.

6. Bugiga FB, Colpo FL, Anzolin D, Kreve S. Restabelecimento da dimensão vertical em paciente com desgastes dentais severos - relato de caso clínico. J Oral Invest. 2016; 5(2):45-52.

7. Cesto FM, Domareski L, Samra APB, Neppelenbroek KH, Campanha NH, Urban VM. Overlay removable partial denture as temporary restoration of vertical dimension of occlusion in a bruxist patient. RGO. 2015;63(1):95-102.

8. Moreno-Hay I, Okeson JP. Does altering the occlusal vertical dimension produce temporomandibular disorders? a literature review. J Oral Rehabil. 2015;42:875-82.

9. Meirelles L, Bavia PF, Vilanova LSR. Aplicações clínicas do enceramento diagnostico na reabilitação oral- uma revisão de literatura. Faculdade de Odontologia de Lins/Unimep. 2013; 23(1):20-5.

10.Bernardo AA, Medeiros MV, Spegel R, Veronez FC, Trauth KGS. Diagnostico e planejamento reabilitador em desdentados: relato de caso. Rev Odontol Univ Cid São Paulo. 2015 mai-ago.; 27(2):142-49.

11.Dantas EM. A importância do restabelecimento da dimensão vertical de oclusão na reabilitação protética. Odonto. 2012;20(40):41-8.

12.Trentin LM et al. Determinação da dimensão vertical de oclusão em prótese total: revisão de literatura e relato de caso. J Oral Invest. 2016; 5(1):50-60.

13.Willis FM. Esthetics of full denture construction. J Amber Dent Assoc. 1930;17:636-41.

14.Rios ACFC, Silva KMG, Sampaio RC, Carvalho EAV, Parente SU. Uso de prótese provisória tipo overlay como recurso de avaliação funcional em indivíduos com alteração da dimensão vertical de oclusão. Odontol Clín Cient. 2016;15(2):135-40.

15.Nagpal A, Parkash H, Bhargava A, Chittaranjan B. Reliability of diferente facial measurements for determination of vertical dimension of occlusion 
in edentulous susing accepted facial dimensions recorded from dentulous subjects. J Indian Prosthodont Soc. 2014;14(3):233-42.

16. Silva MCVS, Carreiro AFP, Bonan RF, Carlo HL, Batista AUD. Reabilitação oclusal em prótese parcial removível provisória tipo "overlay"- relato de caso. Rev Bras Ciên Saúde. 2011;15(4):455-60.

17. Souza JEA, Silva ET, Leles CR. Prótese parcial removível orvelay: fundamentos clínicos e relatos de casos. ROBRAC. 2009;18(47):41-8.

\section{CONFLITO DE INTERESSES}

Os autores declaram não haver conflitos de interesse.

\section{AUTOR PARA CORRESPONDENCIA}

\section{Franklin Barbosa da Silva}

frank_barbosa@hotmail.com

Submetido em 04/09/2018

Aceito em 04/10/2018 